\title{
Impact of processing technique on bronchoalveolar lavage cellular analysis
}

\begin{abstract}
To the Editor:
We read with great interest the study by Bollmann et al. [1] wherein the authors describe the impact of collection protocols and bronchoscope lumen size on bronchoalveolar lavage fluid (BALF) cellular analysis. Their findings highlight the importance of standardising BALF collection protocols in order to meaningfully interpret and compare BALF analysis results, for both clinical and research purposes. As a complement to their study, we would like to emphasise the importance of laboratory methods used to process the collected sample. We present our experience with highly discordant BALF cell counts and differentials, using two processing protocols in our local laboratory.
\end{abstract}

Multiple elements in the laboratory method used for BALF processing, including cytocentrifugation technique and staining, can impact BALF cell differential [2-4]. Published guidelines for BALF processing and interpretation recommend that cytocentrifugation with Giemsa type staining (CY) be used for differential cell count analysis [5]. ThinPrep (TP) technology with Papanicolaou staining is a liquid-based preparation method for cytological specimens which has been adopted by many pathology laboratories as it is automated and results in a clean background with an even distribution of cells on the slide. The Papanicolaou stain used for TP is optimal for detecting atypical and malignant cells and but not for differentiating inflammatory cells [6]. There is a paucity of data comparing the techniques for BALF cellular analysis, and the reported prevalence of either technique in clinical practice is unknown [5]. Until recently, BALF cellular analysis was performed exclusively using TP at our institution, due to the relatively higher cost and resource intensity of CY. As a quality improvement initiative, we compared the BALF cell count differentials obtained using both techniques concurrently during this time of transition from TP to $\mathrm{CY}$ in accordance with current guidelines.

We retrospectively identified BALF samples collected for clinical purposes via flexible bronchoscopy over a 13 month period from four hospitals in Calgary, AB, Canada. Each sample was analysed using both TP and CY methods concurrently, with all samples analysed at a single laboratory.

TP (Cytyc, Marlborough, MA, USA) samples were prepared according to the manufacturer's instructions. The sample was placed into an alcohol-based preservative solution, Cytolyte (Cytyc), centrifuged to obtain cell pellet that was resuspended in Preservcyt (Cytyc), then an even monolayer of cells was collected using a filter. The cells were subsequently transferred to a glass slide, and stained with the Papanicolaou technique. The bronchoalveolar lavage (BAL) differential was performed by a cytopathology technologist and a cytopathologist performed the final review.

For the CY method, initial total white blood cell (WBC) and red blood cell (RBC) counts were analysed using a Sysmex Cell count analyser (Sysmex Canada, Mississauga, ON, Canada). If WBC count was $>10000 \times 10^{6} \mathrm{~L}^{-1}$ or $\mathrm{RBC}>5000000 \times 10^{6} \mathrm{~L}^{-1}$ a dilution was performed to $1000 \times 10^{6} \mathrm{~L}^{-1}$. If the BALF was viscous, a few grains of hyaluronidase were added, mixing well for $10 \mathrm{~min}$; if still viscous, the sample was then incubated at $37^{\circ} \mathrm{C}$ for $5 \mathrm{~min}$, mixed thoroughly, then run through the Sysmex analyser. Two cytospin slides were then prepared using a Shandon CytoSpin 3 cytocentrifuge (Thermo Fisher Scientific, Waltham, MA, USA) at 1000 RPM for $3 \mathrm{~min}$. The slides were stained with Wright-Giemsa stain and the BAL differential was performed by a haematopathology technologist with a haematopathologist performing the final review.

@ERSpublications

BALF cellular analysis results are greatly impacted by processing technique \#AdvancesinILD @ERSTalk http://ow.ly/u9js30i1dUP

Cite this article as: Barss L, Fraser KL, Kelly MM, et al. Impact of processing technique on bronchoalveolar lavage cellular analysis. Eur Respir J 2018; 51: 1701769 [https://doi.org/10.1183/ 13993003.01769-2017]. 
For each sample, cellular composition was categorised according to American Thoracic Society (ATS) standard BAL diagnostic patterns (lymphocytic $>15 \%$; neutrophilic $>3 \%$; eosinophilic $>1 \%$ ) and according to BAL patterns that suggest specific types of interstitial lung disease (ILD; lymphocyte differential count $>50 \%$; neutrophil count $>50 \%$; eosinophil count $>25 \%$ ) [5]. Descriptive comparisons were used to assess the differences in patterns between the two methods. Sensitivities and specificities for TP were calculated using CY values as the reference standard for each specific pattern.

BALF cellular analyses from both methods were available from 28 samples (26 patients; two patients with BAL from two lobes). BALF sampling was undertaken to assess for infection in $9 / 28$ (32\%), airspace disease in $5 / 28(18 \%)$, suspected eosinophilic lung disease in $3 / 28(11 \%)$, malignancy in $1 / 28(3 \%)$, and "other" in 10/28 (36\%).

Classification of BALF according to the analysis method used for standard and ILD specific cellular patterns is shown in the table 1. More samples were classified as an ILD specific pattern (for all cell types) when analysed by CY versus TP, whereas there was no trend in classification by the standard cellular pattern. Cellular pattern was discordant between analysis techniques for a lymphocytic pattern in 5/28 (18\%), neutrophilic pattern in $7 / 28$ (25\%), and eosinophilic pattern in $12 / 28(43 \%)$. There were fewer discordant samples when classified by ILD specific pattern, however, 10/28 samples (36\%) were still discordant by at least one ILD specific pattern. The absolute difference between techniques ranged from $-47 \%$ to $70 \%$, with no consistent directionality. TP had poor sensitivity for ILD specific lymphocytic and eosinophilic patterns ( $0 \%$ for both).

BALF cellular analyses were discordant when TP was compared to CY in a majority of samples for both BAL and ILD specific patterns. TP had very poor sensitivity for ILD specific lymphocytic and eosinophilic patterns. Our findings highlight the impact of BALF processing and analysis techniques, and emphasise that standardised techniques must be broadly implemented. Our findings support previous literature demonstrating BALF processing techniques can impact the BALF cell differential. SALTiNI et al. [2] compared BALF cell differential counts from a cytocentrifuge versus non-cytocentrifuge technique and found cytocentrifugation was consistently associated with a loss in lymphocyte percentage. A more recent study demonstrated variations within the cytocentrifugation conditions can also impact the BALF cell differential [3]

BALF cellular analysis is proposed as an informative clinical tool in the diagnosis of certain pulmonary diseases, most commonly ILDs, such as hypersensitivity pneumonitis, nonspecific interstitial pneumonia and eosinophilic pneumonia [5]. However, for BALF to contribute meaningful pathobiological information, results must be accurate, with accuracy dependent upon processing technique. An informal survey (personal communications, K.A. Johannson) of ILD practitioners from different centres suggests that many clinicians are unfamiliar with the BALF processing techniques in use at their institution. Clinicians should enquire as to their local BALF processing and analytic techniques if BALF cellular analyses are being used for clinical or research purposes, and aim to implement the currently recommended technique.

Our data have important limitations, including a small sample size and retrospective study design. We compared only TP results with the ATS standard, CY, thus the discordant results cannot be generalised to

TABLE 1 Classification and performance by analysis method for bronchoalveolar lavage cellular pattern

\begin{tabular}{lcccc} 
& TP $(\mathbf{n}=28)$ & CY (n=28) & \multicolumn{2}{c}{$\begin{array}{c}\text { Sensitivity and specificity of TP } \\
\text { for cellular patterns (versus CY) }\end{array}$} \\
\cline { 3 - 4 } & & & Specificity \\
\hline Standard cellular pattern & & & & \\
$\quad$ Neutrophilic $(>3 \%)$ & $27(96 \%)$ & $22(79 \%)$ & $21 / 22(95 \%)$ & $0 / 6(0 \%)$ \\
Lymphocytic $(>15 \%)$ & $5(18 \%)$ & $10(36 \%)$ & $5 / 10(50 \%)$ & $18 / 18(100 \%)$ \\
Eosinophilic $(>1 \%)$ & $18(64 \%)$ & $14(50 \%)$ & $10 / 14(71 \%)$ & $6 / 14(43 \%)$ \\
ILD specific pattern & $7(25 \%)$ & $8(29 \%)$ & $7 / 8(87.5 \%)$ & $20 / 20(100 \%)$ \\
Neutrophilic $(>50 \%)$ & $1(4 \%)$ & $3(11 \%)$ & $0 / 3(0 \%)$ & $24 / 25(96 \%)$ \\
Lymphocytic $(>50 \%)$ & 0 & $4(14 \%)$ & $0 / 4(0 \%)$ & $24 / 24(100 \%)$ \\
Eosinophilic $(>25 \%)$ & & & & \\
\hline
\end{tabular}

CY: cytocentrifugation with Giemsa type staining; TP: ThinPrep technology; ILD: interstitial lung disease. 
other preparation and staining methods. Data collection was performed primarily for local quality assessment so we cannot account for patient-specific factors that can affect BALF, such as smoking status or BALF collection techniques. However, we compared the two methods of analysis within the same sample so it is unlikely that these factors affected our intra-sample discordance.

We hope that our data, combined with that of BolLmann et al. [1], will inform the potential sources of discrepancy between and within centres when it comes to the role of BALF analysis, a timely topic given the widespread interest in diseases such as hypersensitivity pneumonitis. Accurate results are critical for meaningful applications of BALF analysis, and we encourage ongoing collaborative efforts to standardise BALF collection, processing and analysis to guide research and to optimise patient care.

Leila Barss ${ }^{1}$, Kristin L. Fraser ${ }^{1}$, Margaret M. Kelly ${ }^{2}$ and Kerri A. Johannson ${ }^{1}$

${ }^{1}$ Dept of Medicine, University of Calgary, Calgary, AB, Canada. ${ }^{2}$ Dept of Pathology and Laboratory Medicine, University of Calgary, Calgary, AB, Canada.

Correspondence: Kerri A. Johannson, 4448 Front Street SE, Calgary, AB T3M-1M4, Canada.

E-mail: kerri.johannson@ahs.ca

Received: Aug 292017 | Accepted after revision: Jan 102018

Conflict of interest: K.A. Johannson has received personal fees and non-financial support from Hoffman-La Roche Ltd and Boehringer-Ingelheim, and grants from UCB Biopharma SPRL and CHEST Foundation, outside the submitted work.

\section{References}

1 Bollmann BA, Seeliger B, Drick N, et al. Cellular analysis in bronchoalveolar lavage: inherent limitations of current standard procedure. Eur Respir J 2017; 49: 1601844.

2 Saltini C, Hance AJ, Ferrans VJ, et al. Accurate quantification of cells recovered by bronchoalveolar lavage. Am Rev Respir Dis 1984; 130: 650-658.

3 De Brauwer EI, Jacobs JA, Nieman F, et al. Cytocentrifugation conditions affecting the differential cell count in bronchoalveolar lavage fluid. Anal Quant Cytol Histol 2000; 22: 416-422.

4 De Brauwer EI, Jacobs JA, Nieman F, et al. Bronchoalveolar lavage fluid differential cell count. How many cells should be counted? Anal Quant Cytol Histol 2002; 24: 337-341.

5 Meyer KC, Raghu G, Baughman RP, et al. An official American Thoracic Society clinical practice guideline: the clinical utility of bronchoalveolar lavage cellular analysis in interstitial lung disease. Am J Respir Crit Care Med 2012; 185: 1004-1014.

6 Baughman RP. Technical aspects of bronchoalveolar lavage: recommendations for a standard procedure. Semin Respir Crit Care Med 2007; 28: 475-485. 